\title{
Supporting Information: QM/MM Studies on Thermally Activated Delayed Fluorescence of a Dicopper Complex in the Solid State
}

Ling-Ya Peng, Wen-kai Chen, Zi-Wen Li, Yuan-Jun Gao*, Ganglong Cui*

Key Laboratory of Theoretical and Computational Photochemistry, Ministry of Education, Chemistry College, Beijing Normal University, Beijing 100875, P. R. China

Email: yuanjun.gao@mail.bnu.edu.cn and ganglong.cui@bnu.edu.cn

\section{Table of Contents}

\section{$\underline{\text { I Figures }}$}

I. Figure Si. The ONIOM model in which the innermost molecule and two counter ions PF6- are chosen as the QM region and the surrounding 2I molecules are done as the MM region.

2. Figure S2. Overlap of the optimized So structures (the QM region) in the two ONIOM models with different surrounding MM molecules (I4 and 2I molecules).

3. Figure $S_{3}$. Overlap of optimized $S_{o}, S_{I}$, and $T_{I}$ structures in crystal.

4. Figure $\mathrm{S}_{4}$. (Left) TD-DFT calculated single-point energies at the $\mathrm{S}_{\mathrm{o}}, \mathrm{S}_{\mathrm{I}}$ and $\mathrm{T}_{\mathrm{I}}$ minima in crystal; (Right) the relevant frontier molecular orbitals involved.

5. Figure $\mathrm{S}_{5}$. Selected molecular orbitals of dicopper complex at $\mathrm{S}_{0}$ minima in crystal.

6. Figure S6. Duschinsky matrix elements of dicopper complexes in ISC and rISC processes. 


\section{$\underline{\text { II Tables }}$}

7. Table Si. Calculated Absorption Energies Using the Initial (I4 Molecules in MM region) and Tested ONIOM Models (2I Molecules in MM region).

8. Table S2. Calculated Absorption, Fluorescence and Phosphorescence Energies and the Energy Gaps between the $S_{I}$ and $T_{I}$ States Using Different Functionals.

9. Table $S_{3}$. Selected Geometric Parameters of Dicopper Complex at the $S_{0}, S_{1}$ and $\mathrm{T}_{\mathrm{I}}$ minima.

Io. Table S4. Calculated Vertical Excitation Energies, Wavelength, Oscillator Strengths, and Main Configurations of Dicopper Complex in Crystal.

II. Table S5. Calculated Vertical Emission Energies, Wavelength, Oscillator Strengths, and Electronic Configurations at the $\mathrm{S}_{\mathrm{I}}$ and $\mathrm{T}_{\mathrm{I}}$ Minima in Crystal.

12. Table S6. Calculated Spin-Orbit Coupling Matrix Elements in (Reverse) Intersystem Crossing Processes.

13. Table S7. Temperature Dependence of (Reverse) Intersystem Crossing Rates.

14. Table S8. Calculated (Reverse) Intersystem Crossing Rates without Duschinsky Effect.

\section{Cartesian Coordinates of Optimized Structures of QM}




\section{$\underline{\text { I Figures }}$}

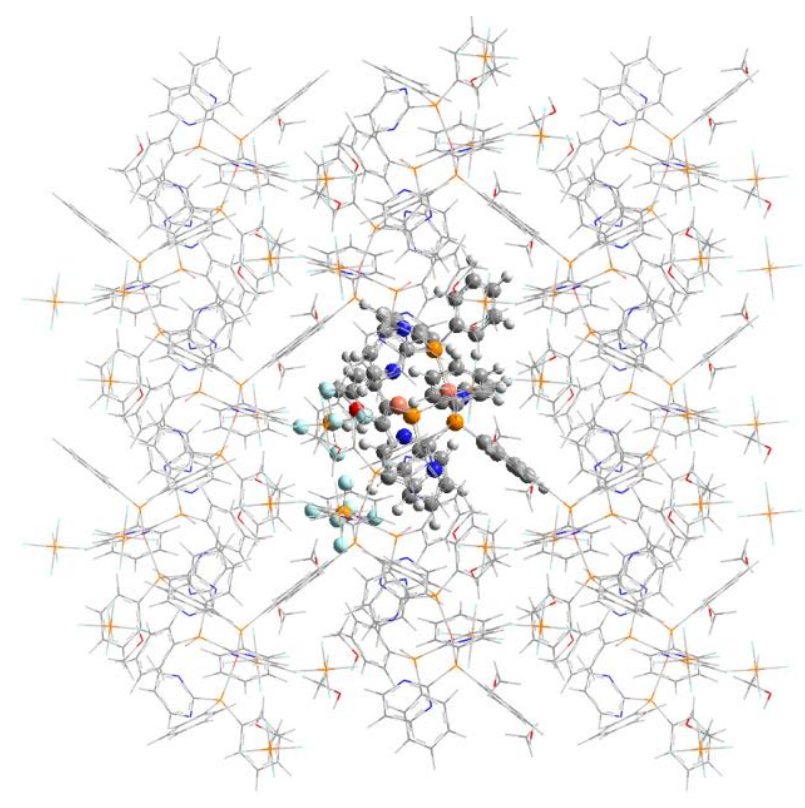

Figure Sr. The ONIOM model in which the innermost molecule and two counter ions PF6- are chosen as the QM region and the surrounding 21 molecules are done as the MM region.

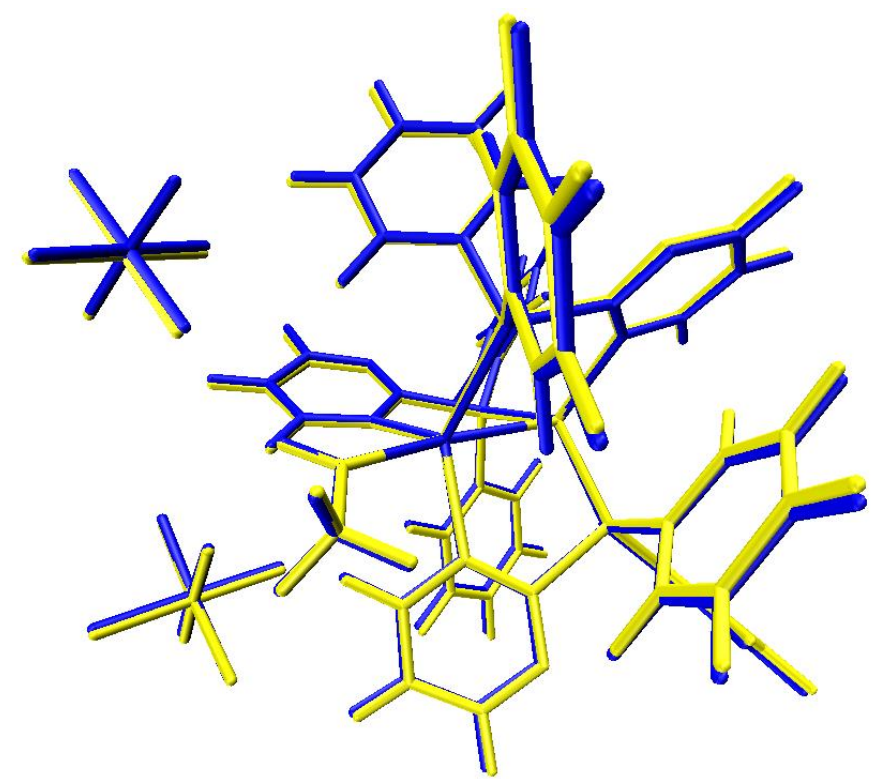

Figure S2. Overlap of the optimized So structures (the QM region) in the two ONIOM models with different surrounding MM molecules (I4 and 2I molecules). 


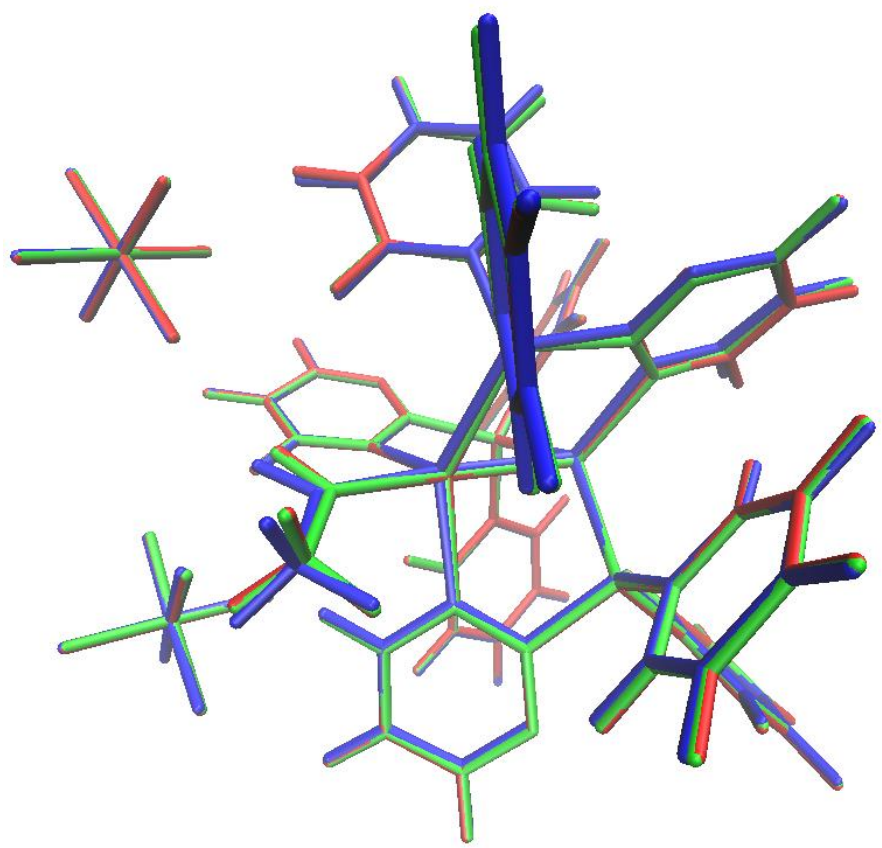

Figure $S_{3}$. Overlap of optimized $S_{0}$ (blue), $S_{I}$ (red), and $T_{I}$ (green) structures in crystal.
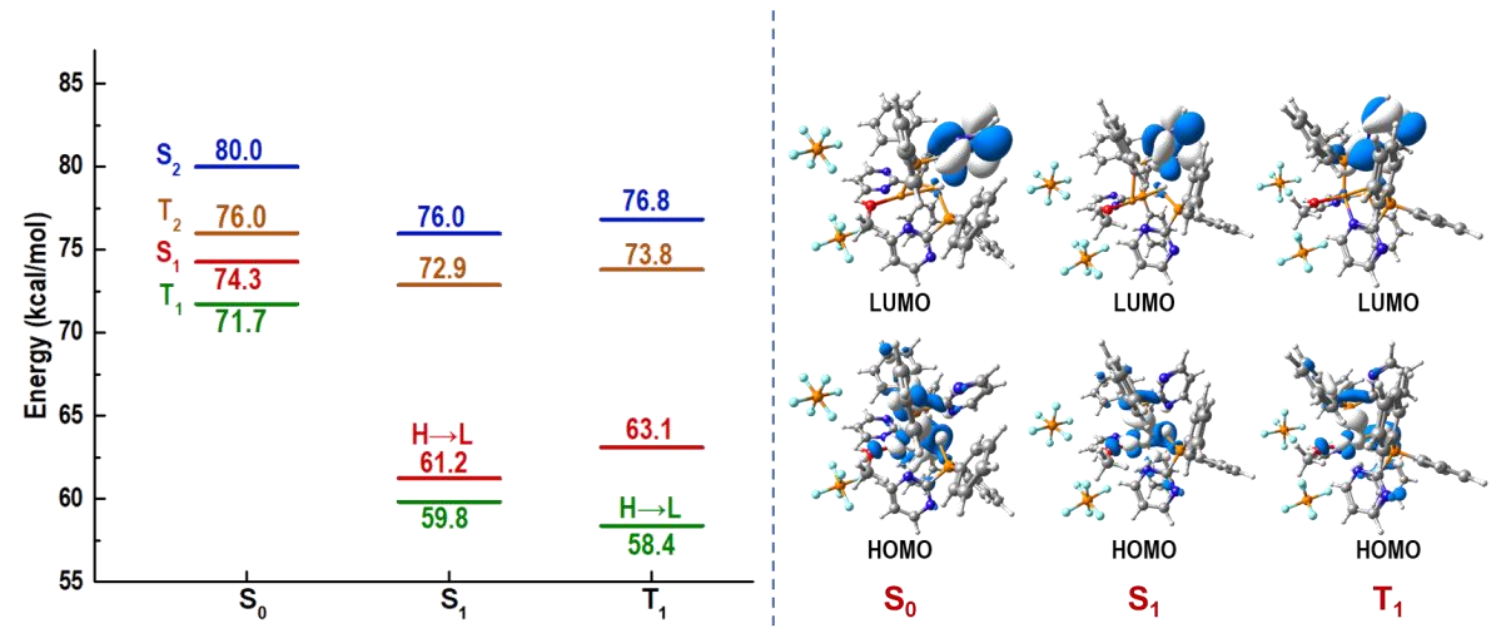

Figure $S_{4}$. (Left) TD-DFT calculated vertical energies at the $S_{o}, S_{I}$ and $T_{I}$ minima in crystal; (Right) the relevant frontier molecular orbitals involved. 


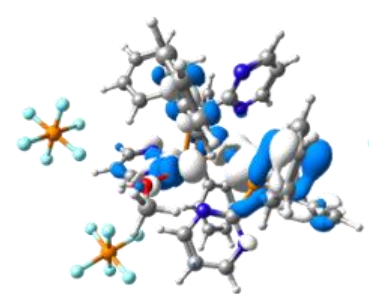

HOMO-3

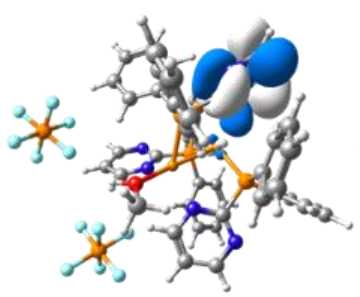

LUMO

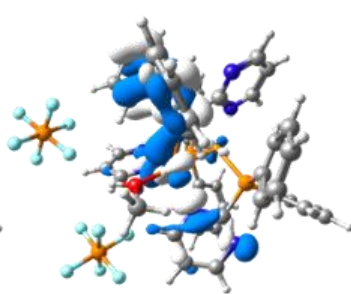

HOMO-2

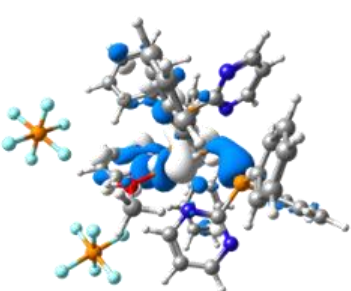

HOMO-1

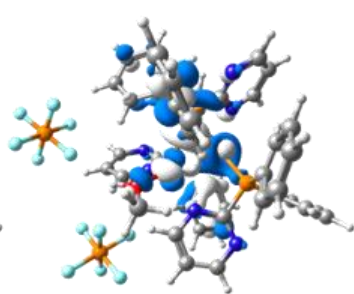

HOMO

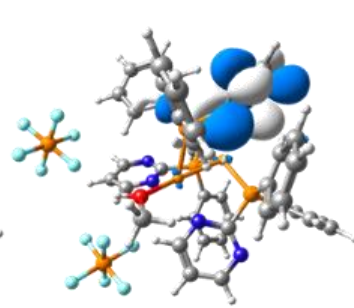

LUMO+1

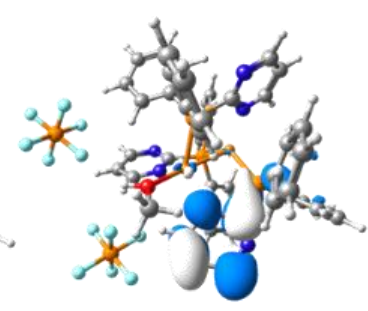

LUMO+2

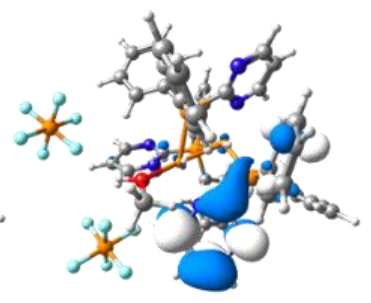

LUMO+3

Figure $\mathrm{S}_{5}$. Selected molecular orbitals of the dicopper complex at $\mathrm{S}_{0}$ minima in crystal.
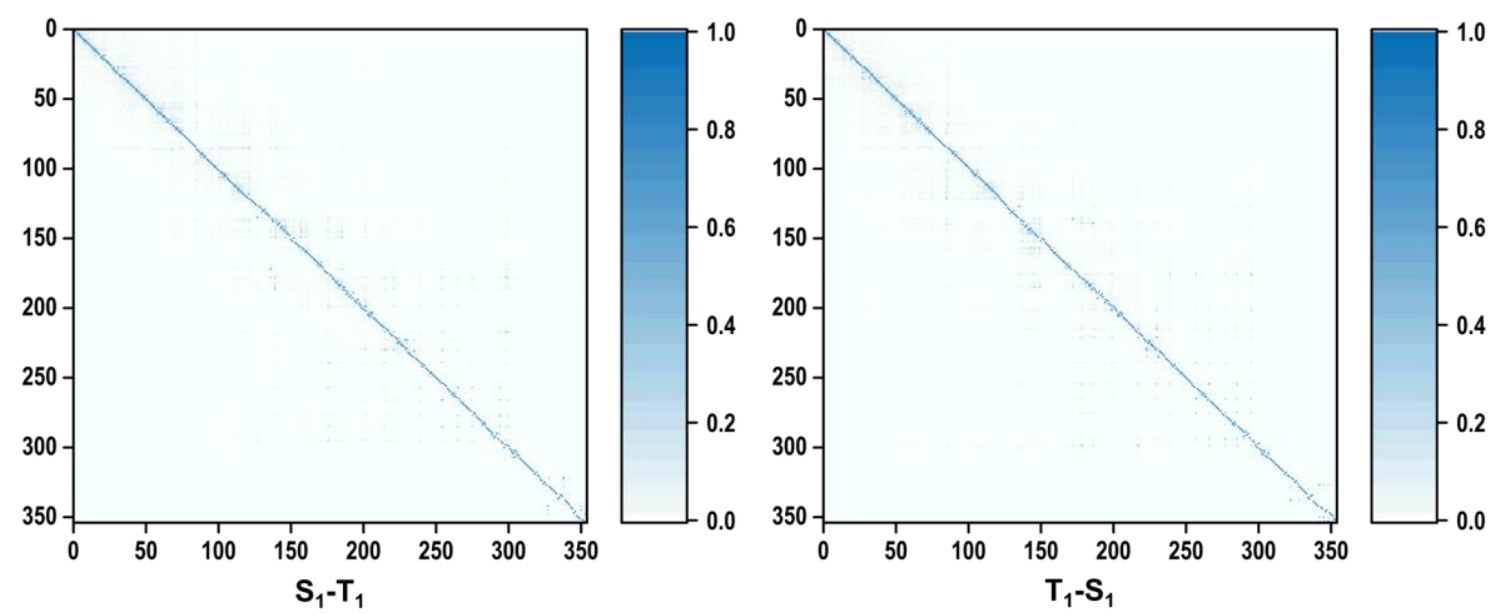

Figure S6. Duschinsky matrices of the dicopper complex in the ISC (left) and rISC (right) processes. 


\section{Tables}

Table Si. Calculated Absorption Energies Using the Initial (I4 Molecules in MM region) and Tested ONIOM Models (2I Molecules in MM region).

\begin{tabular}{|c|c|c|c|c|c|c|}
\hline & \multicolumn{3}{|c|}{ Initial ONIOM Model } & \multicolumn{3}{|c|}{ Tested ONIOM model } \\
\hline & $\begin{array}{c}E_{\perp} \\
(\mathrm{eV})\end{array}$ & $\begin{array}{c}\mathrm{E}_{\perp} \\
(\mathrm{kcal} / \mathrm{mol})\end{array}$ & $\begin{array}{c}\lambda \\
(\mathrm{nm})\end{array}$ & $\begin{array}{c}E_{\perp} \\
(e V)\end{array}$ & $\begin{array}{c}\mathrm{E}_{\perp} \\
(\mathrm{kcal} / \mathrm{mol})\end{array}$ & $\begin{array}{c}\lambda \\
(\mathrm{nm})\end{array}$ \\
\hline So $\rightarrow S_{I}$ & 3.22 & 74.3 & 385 & 3.23 & 74.5 & 384 \\
\hline $\mathrm{So}_{\mathrm{S}} \rightarrow \mathrm{S}_{2}$ & 3.47 & 80.I & 357 & 3.47 & 80.1 & 357 \\
\hline $\mathrm{So}_{\mathrm{S}} \rightarrow \mathrm{S}_{3}$ & $3.6 \mathrm{I}$ & 83.4 & 343 & 3.63 & 83.6 & 342 \\
\hline
\end{tabular}

Table S2. Calculated Absorption, Fluorescence and Phosphorescence Energies (Abs., FL., PL., kcal/mol) and the Energy Gaps between the $\mathrm{S}_{\mathrm{I}}$ and $\mathrm{T}_{\mathrm{I}}$ States $\left(\Delta \mathrm{E}_{\mathrm{ST}}\right.$, kcal/mol) Using Different Functionals.

\begin{tabular}{|c|c|c|c|c|c|c|}
\hline & PBEo & B 3 LYP & Mo62X & CAM-B3LYP & wB97XD & Exp. \\
\hline Abs. & 74.3 & 69.6 & 97.6 & 94.8 & 96.2 & - \\
\hline FL. & 52.8 & 48.3 & 78.6 & 74.0 & 76.2 & 56.1 \\
\hline PL. & 48.9 & 46.2 & 67.5 & 61.6 & 63.2 & 53.9 \\
\hline$\Delta \mathrm{E}_{\text {ST }}$ & 2.8 & I.0 & 9.4 & II.I & 11.7 & 2.3 \\
\hline
\end{tabular}


Table S3. Selected Geometric Parameters of Dicopper Complex at the $\mathrm{S}_{\mathrm{o}}, \mathrm{S}_{\mathrm{I}}$ and $\mathrm{T}_{\mathrm{I}}$ minima (Bond Length in $\AA$ and Angles in ${ }^{\circ}$ ).

\begin{tabular}{|c|c|c|c|c|}
\hline & Exp. & $\mathrm{S}_{\mathrm{o}}$ & $S_{I}$ & $\mathrm{~T}_{\mathrm{I}}$ \\
\hline $\mathrm{Cur}-\mathrm{O}$ & 2.201 & 2.144 & $2.08 \mathrm{I}$ & 2.085 \\
\hline $\mathrm{CuI}-\mathrm{Cu} 2$ & 2.630 & 2.621 & 2.552 & 2.568 \\
\hline $\mathrm{CuI}-\mathrm{NI}_{\mathrm{I}}$ & $2.13 \mathrm{I}$ & 2.179 & 2.227 & 2.208 \\
\hline $\mathrm{CuI}-\mathrm{N}_{2}$ & 2.068 & 2.122 & 2.108 & $2.11 I$ \\
\hline $\mathrm{CuI}_{\mathrm{I}}-\mathrm{P}_{\mathrm{I}}$ & 2.230 & 2.327 & 2.378 & 2.375 \\
\hline $\mathrm{Cu}_{2}-\mathrm{P}_{2}$ & 2.213 & 2.259 & 2.275 & 2.269 \\
\hline $\mathrm{Cu}_{2}-\mathrm{P}_{3}$ & 2.254 & 2.297 & 2.320 & 2.313 \\
\hline $\mathrm{Cu}_{2}-\mathrm{N}_{3}$ & 2.002 & 2.053 & 2.005 & I.986 \\
\hline $\mathrm{O}-\mathrm{CuI}-\mathrm{Cu} 2$ & 169.5 & 169.6 & I68.7 & I68.2 \\
\hline $\mathrm{O}-\mathrm{CuI}-\mathrm{NI}$ & $94 \cdot 3$ & 94.0 & 89.7 & 90.6 \\
\hline $\mathrm{O}-\mathrm{CuI}_{\mathrm{I}} \mathrm{N}_{2}$ & 89.0 & 88.2 & 87.7 & 87.5 \\
\hline $\mathrm{O}-\mathrm{CuI}-\mathrm{Pr}$ & IO2.I & I03.8 & IOI.2 & 101.5 \\
\hline $\mathrm{Cu}-\mathrm{Cu}_{2}-\mathrm{P}_{2}$ & 86.5 & 85.5 & 81.9 & 82.1 \\
\hline $\mathrm{CuI}-\mathrm{Cu}_{2}-\mathrm{P}_{3}$ & 83.5 & 86.4 & 84.4 & $84 . I$ \\
\hline $\mathrm{Cu}-\mathrm{Cu}_{2}-\mathrm{N}_{3}$ & 108.4 & III.9 & II 4.4 & 115.4 \\
\hline $\mathrm{CuI}_{\mathrm{I}}-\mathrm{N}_{1}-\mathrm{N}_{2}-\mathrm{PI}_{\mathrm{I}}$ & -8.5 & -9.5 & -5.8 & -6.3 \\
\hline $\mathrm{Cu}_{2}-\mathrm{P}_{2}-\mathrm{P}_{3}-\mathrm{N}_{3}$ & -5.5 & -8.4 & -5.0 & -5.6 \\
\hline $\mathrm{O}-\mathrm{CuI}-\mathrm{N}_{1}-\mathrm{N}_{2}$ & -89.5 & -89.1 & -87.5 & -87.6 \\
\hline $\mathrm{O}-\mathrm{Cu}_{2}-\mathrm{P}_{2}-\mathrm{P}_{3}$ & -79.0 & $-8 \mathrm{I} .0$ & -75.9 & -75.6 \\
\hline
\end{tabular}

Table S4. Calculated Vertical Excitation Energies $\left(E_{\perp} / \mathrm{eV}\right)$, Wavelength $(\lambda / \mathrm{nm})$, Oscillator Strengths ( $f$ ), and Main Configurations of Dicopper Complex in Crystal.

\begin{tabular}{|c|c|c|c|c|}
\hline States & $\mathrm{E}_{\perp}(\mathrm{eV})$ & $\boldsymbol{\lambda}(\mathrm{nm})$ & $\boldsymbol{f}$ & Configurations \\
\hline $\mathrm{S}_{\mathrm{I}}$ & 3.22 & 385 & 0.0023 & HOMO $\rightarrow$ LUMO $(96.8 \%)$ \\
\hline $\mathrm{S}_{\mathbf{2}}$ & 3.47 & 357 & 0.0179 & HOMO $\rightarrow$ LUMO+I $(94.8 \%)$ \\
\hline $\mathrm{S}_{3}$ & $3.6 \mathrm{I}$ & 343 & 0.0134 & HOMO-I $\rightarrow$ LUMO $(90.0 \%)$ \\
\hline $\mathrm{S}_{4}$ & 3.72 & 333 & 0.0150 & HOMO $\rightarrow$ LUMO+2 (81.0\%) \\
\hline $\mathrm{S}_{5}$ & 3.78 & 328 & 0.0310 & $\begin{array}{c}\text { HOMO-3 } \rightarrow \text { LUMO (49.6\%) } \\
\text { HOMO-2 } \rightarrow \text { LUMO (20.5\%) } \\
\text { HOMO-I } \rightarrow \text { LUMO+I (II.0\%) }\end{array}$ \\
\hline
\end{tabular}


Table S5. Calculated Vertical Emission Energies $\left(\mathrm{E}_{\perp} / \mathrm{eV}, \mathrm{kcal} / \mathrm{mol}\right), \mathrm{W}$ avelength $(\lambda / \mathrm{nm})$, Oscillator Strengths $(f)$, and Electronic Configurations at the $S_{I}$ and $T_{I}$ Minima.

\begin{tabular}{|c|c|c|c|c|c|}
\hline & \multicolumn{5}{|c|}{$\mathrm{S}_{\mathrm{I}}$} \\
\hline & \multicolumn{2}{|r|}{$E_{\perp}$} & $\lambda$ & \multirow[b]{2}{*}{$f$} & \multirow{2}{*}{ Configurations } \\
\hline & $(\mathrm{eV})$ & $(\mathrm{kcal} / \mathrm{mol})$ & $(\mathrm{nm})$ & & \\
\hline TD & 2.29 & 52.8 & $54 \mathrm{I}$ & 0.0017 & $\mathrm{H}<-\mathrm{L}(99.0 \%)$ \\
\hline \multirow[t]{4}{*}{ Exp. } & 2.43 & 56.1 & 510 & & \\
\hline & \multicolumn{5}{|c|}{$\mathrm{T}_{\mathrm{I}}$} \\
\hline & \multicolumn{2}{|r|}{$\mathrm{E}_{\perp}$} & $\lambda$ & \multirow{2}{*}{$f$} & \multirow{2}{*}{ Configurations } \\
\hline & $(\mathrm{eV})$ & $(\mathrm{kcal} / \mathrm{mol})$ & $(\mathrm{nm})$ & & \\
\hline $\mathrm{TD}$ & 2.12 & 48.9 & 584 & 0.0000 & $\mathrm{H}<-\mathrm{L}(94.0 \%)$ \\
\hline Exp. & 2.34 & 53.9 & 530 & & \\
\hline
\end{tabular}

Table S6. Calculated Spin-Orbit Coupling Matrix Elements (SOCMEs/ $\mathrm{cm}^{-1}$ ) in (Reverse) Intersystem Crossing Processes.

\begin{tabular}{|c|c|c|}
\hline & ISC $\left(\mathrm{S}_{\mathrm{I}} \rightarrow \mathrm{T}_{\mathrm{I}}\right)$ & $\operatorname{rISC}\left(\mathrm{T}_{\mathrm{I}} \rightarrow \mathrm{S}_{\mathrm{I}}\right)$ \\
\hline SOCMEs $\left(\mathrm{cm}^{-\mathrm{I}}\right)$ & 2.06 & $8.6 \mathrm{I}$ \\
\hline
\end{tabular}

Table S7. Temperature Dependence of (Reverse) Intersystem Crossing Rates $\left(\mathrm{s}^{-1}\right)$.

\begin{tabular}{|c|c|c|c|c|c|c|}
\hline & $77 \mathrm{~K}$ & $100 \mathrm{~K}$ & $150 \mathrm{~K}$ & $200 \mathrm{~K}$ & $250 \mathrm{~K}$ & $300 \mathrm{~K}$ \\
\hline$k_{\mathrm{ISC}}\left(\mathrm{S}_{\mathrm{r}-\mathrm{Tr}}\right)$ & $7.28 \mathrm{E}+08$ & $7.44 \mathrm{E}+08$ & $7.52 \mathrm{E}+08$ & $7.45 \mathrm{E}+08$ & $7.37 \mathrm{E}+08$ & $7.31 \mathrm{E}+08$ \\
\hline$k_{\mathrm{rISC}}\left(\mathrm{Tr}_{\mathrm{r}-\mathrm{S}}\right)$ & $10.2 \mathrm{I}$ & $\mathrm{I} .19 \mathrm{E}+03$ & $2.2 \mathrm{I} \mathrm{E}+05$ & $2.93 \mathrm{E}+06$ & $1.36 \mathrm{E}+07$ & $3.76 \mathrm{E}+07$ \\
\hline
\end{tabular}

Table S8. Calculated (Reverse) Intersystem Crossing Rates $\left(\mathrm{s}^{-1}\right)$ without Duschinsky Effect.

\begin{tabular}{|c|c|c|}
\hline & $77 \mathrm{~K}$ & $300 \mathrm{~K}$ \\
\hline$k_{\mathrm{ISC}}\left(\mathrm{S}_{\mathrm{I}}-\mathrm{Tr}_{\mathrm{r}}\right)$ & $5.66 \mathrm{E}+08$ & $6.58 \mathrm{E}+08$ \\
\hline$k_{\mathrm{rISC}}\left(\mathrm{Tr}_{\mathrm{r}}-\mathrm{S}_{\mathrm{r}}\right)$ & 8.06 & $3.70 \mathrm{E}+07$ \\
\hline
\end{tabular}




\section{Cartesian Coordinates of Optimized Structures of QM}

\begin{tabular}{|c|c|c|c|}
\hline $\begin{array}{l}S_{0} \cdot x \\
\text { II } 8 \\
\text { sym }\end{array}$ & y cI & & \\
\hline $\mathrm{Cu}$ & I.I61762000 & I. 402428000 & 0.605584000 \\
\hline $\mathrm{Cu}$ & -I.371057000 & 2.009555000 & 0.311815000 \\
\hline $\mathrm{P}$ & 0.975957000 & 2.716589000 & -I.306I25000 \\
\hline $\mathrm{N}$ & -I.7096180oo & 3.058810000 & $-\mathrm{I} .420114000$ \\
\hline $\mathrm{N}$ & -0.633928000 & 3.172248000 & -3.522877000 \\
\hline $\mathrm{C}$ & -0.625640000 & 2.988908000 & -2.209956000 \\
\hline $\mathrm{C}$ & -2.872183000 & 3.411422000 & -I.9756500oo \\
\hline $\mathrm{H}$ & -3.729354000 & 3.466635000 & -I.318065000 \\
\hline $\mathrm{C}$ & -2.969671000 & 3.668198000 & -3.331780000 \\
\hline $\mathrm{H}$ & -3.908973000 & 3.953361000 & -3.789600000 \\
\hline $\mathrm{C}$ & -І.80442100o & 3.498140000 & -4.071356000 \\
\hline $\mathrm{H}$ & -I.808051000 & 3.633884000 & -5.143103000 \\
\hline $\mathrm{C}$ & I. 388354000 & 4.464983000 & -0.996422000 \\
\hline $\mathrm{C}$ & 0.680058000 & 5.502724000 & -1.615906000 \\
\hline $\mathrm{H}$ & -0.127822000 & $5 \cdot 300684000$ & -2.309863000 \\
\hline $\mathrm{C}$ & I.0198660oo & 6.824705000 & -I.369947000 \\
\hline $\mathrm{H}$ & 0.446836000 & 7.606042000 & -I.853452000 \\
\hline $\mathrm{C}$ & 2.081952000 & 7.127299000 & -0.525542000 \\
\hline $\mathrm{H}$ & 2.358382000 & 8.162624000 & -0.355305000 \\
\hline $\mathrm{C}$ & 2.795471000 & 6.102647000 & 0.091940000 \\
\hline $\mathrm{H}$ & 3.622443000 & 6.318804000 & 0.755156000 \\
\hline C & 2.446901000 & 4.773111000 & -0.132515000 \\
\hline $\mathrm{H}$ & 3.022596000 & 3.990569000 & 0.354065000 \\
\hline $\mathrm{C}$ & 2.028110000 & 2.145851000 & -2.667834000 \\
\hline $\mathrm{C}$ & 2.680913000 & 3.038053000 & -3.521501000 \\
\hline $\mathrm{H}$ & 2.659470000 & 4.102838000 & -3.313776000 \\
\hline $\mathrm{C}$ & 3.358577000 & 2.555897000 & -4.636269000 \\
\hline $\mathrm{H}$ & 3.848265000 & 3.255448000 & -5.304814000 \\
\hline $\mathrm{C}$ & 3.393791000 & I.I84097000 & -4.890416000 \\
\hline $\mathrm{H}$ & 3.904012000 & 0.800128000 & -5.766779000 \\
\hline $\mathrm{C}$ & 2.750080000 & 0.294107000 & -4.033340000 \\
\hline $\mathrm{H}$ & 2.760213000 & -0.768952000 & -4.244251000 \\
\hline C & 2.070002000 & 0.772786000 & -2.918567000 \\
\hline $\mathrm{H}$ & I. 551933000 & 0.082528000 & -2.257213000 \\
\hline $\mathrm{P}$ & -I.7080380oo & -0.225154000 & -o.IoI4IIOoo \\
\hline $\mathrm{N}$ & 0.567123000 & -0.523001000 & I. 271904000 \\
\hline $\mathrm{N}$ & -0.943865000 & -2.350462000 & I.454297000 \\
\hline C & -0.578578000 & -I.I63205000 & 0.996306000 \\
\hline C & I. 3861420000 & -I.107242000 & 2.153732000 \\
\hline
\end{tabular}




\begin{tabular}{|c|c|c|c|}
\hline $\mathrm{H}$ & 2.314327000 & -0.600724000 & 2.372871000 \\
\hline $\mathrm{C}$ & I.039781000 & -2.297971000 & 2.774515000 \\
\hline $\mathrm{H}$ & I.693319000 & -2.698560000 & 3.533613000 \\
\hline $\mathrm{C}$ & -0.125551000 & -2.917374000 & 2.350755000 \\
\hline $\mathrm{H}$ & -0.421711000 & -3.894160000 & 2.727550000 \\
\hline $\mathrm{C}$ & -I.2116660oo & -0.770592000 & -I.760283000 \\
\hline $\mathrm{C}$ & -0.409091000 & -I.893925000 & -I.977392000 \\
\hline $\mathrm{H}$ & -o.14018400o & -2.523878000 & -I.137232000 \\
\hline $\mathrm{C}$ & 0.034058000 & -2.196937000 & -3.261617000 \\
\hline $\mathrm{H}$ & 0.647042000 & -3.072719000 & -3.438069000 \\
\hline $\mathrm{C}$ & -0.304686000 & -I.372103000 & -4.329080000 \\
\hline $\mathrm{H}$ & 0.099393000 & -1.5663550000 & -5.314109000 \\
\hline $\mathrm{C}$ & -I.I43417000 & -0.282372000 & -4.122377000 \\
\hline $\mathrm{H}$ & $-\mathrm{I} .418029000$ & 0.361120000 & -4.947203000 \\
\hline $\mathrm{C}$ & -І.604986000 & 0.013048000 & -2.848504000 \\
\hline $\mathrm{H}$ & -2.266571000 & 0.860431000 & -2.697171000 \\
\hline $\mathrm{C}$ & -3.333878000 & -0.94011900o & 0.209138000 \\
\hline $\mathrm{C}$ & -3.823233000 & -2.051469000 & -0.480749000 \\
\hline $\mathrm{H}$ & -3.215100000 & -2.532269000 & -I.239786000 \\
\hline $\mathrm{C}$ & -5.086136000 & -2.542536000 & -0.175353000 \\
\hline $\mathrm{H}$ & -5.474016000 & -3.412872000 & -0.695462000 \\
\hline $\mathrm{C}$ & -5.847870000 & -I.917563000 & 0.809282000 \\
\hline $\mathrm{H}$ & -6.827435000 & -2.302081000 & I.04843100o \\
\hline $\mathrm{C}$ & -5.353775000 & -0.823896000 & 1.511527000 \\
\hline $\mathrm{H}$ & -5.939871000 & -0.359893000 & 2.297765000 \\
\hline $\mathrm{C}$ & -4.090768000 & -0.334313000 & I.213641000 \\
\hline $\mathrm{H}$ & -3.671186000 & 0.491418000 & I.780832000 \\
\hline $\mathrm{P}$ & -I.I633080oo & 3.068271000 & 2.296220000 \\
\hline $\mathrm{N}$ & I.4611780oo & 2.511133000 & 2.457207000 \\
\hline $\mathrm{N}$ & 0.768909000 & 4.489333000 & 3.586 II40oo \\
\hline $\mathrm{C}$ & 0.552097000 & 3.423239000 & 2.841429000 \\
\hline $\mathrm{C}$ & 2.674815000 & 2.626549000 & 3.014225000 \\
\hline $\mathrm{H}$ & 3.398834000 & I.857666ooo & 2.795915000 \\
\hline $\mathrm{C}$ & 3.001343000 & 3.696835000 & 3.833395000 \\
\hline $\mathrm{H}$ & 4.003186000 & 3.805815000 & 4.219919000 \\
\hline $\mathrm{C}$ & 2.022181000 & 4.653341000 & 4.032467000 \\
\hline $\mathrm{H}$ & 2.241032000 & 5.574919000 & 4.558267000 \\
\hline $\mathrm{C}$ & -2.034456000 & 4.637931000 & 2.490112000 \\
\hline $\mathrm{C}$ & -2.176619000 & 5.411945000 & I.332506000 \\
\hline $\mathrm{H}$ & $-\mathrm{I} .803203000$ & 5.030460000 & 0.384881000 \\
\hline C & -2.749226000 & 6.677482000 & I.406605000 \\
\hline $\mathrm{H}$ & -2.823327000 & 7.293259000 & 0.514716000 \\
\hline $\mathrm{C}$ & -3.219793000 & 7.149442000 & 2.628293000 \\
\hline $\mathrm{H}$ & -3.678710000 & 8.131506000 & 2.690297000 \\
\hline
\end{tabular}




\begin{tabular}{|c|c|c|c|}
\hline C & -3.098576000 & 6.369336000 & 3.778252000 \\
\hline $\mathrm{H}$ & -3.460624000 & 6.745918000 & 4.729994000 \\
\hline C & -2.475700000 & 5.129129000 & 3.721854000 \\
\hline $\mathrm{H}$ & -2.315217000 & 4.554146000 & 4.627758000 \\
\hline $\mathrm{C}$ & -I.763780ooo & I. 862701000 & 3.529658000 \\
\hline $\mathrm{C}$ & -0.917223000 & 0.815682000 & 3.914586000 \\
\hline $\mathrm{H}$ & 0.131245000 & 0.823308000 & 3.634597000 \\
\hline $\mathrm{C}$ & -I.4044660oo & -0.227132000 & 4.693037000 \\
\hline $\mathrm{H}$ & -0.730459000 & -I.022076000 & 4.994031000 \\
\hline $\mathrm{C}$ & -2.738766000 & -0.248987000 & 5.090043000 \\
\hline $\mathrm{H}$ & -3.096130000 & -I.071494000 & 5.699478000 \\
\hline $\mathrm{C}$ & -3.586463000 & 0.785638000 & 4.701043000 \\
\hline $\mathrm{H}$ & -4.633946000 & 0.789571000 & 4.992031000 \\
\hline $\mathrm{C}$ & -3.105112000 & I.836210000 & 3.922393000 \\
\hline $\mathrm{H}$ & -3.773389000 & 2.638260000 & 3.624487000 \\
\hline$P$ & 6.028166000 & 4.854319000 & 2.138486000 \\
\hline $\mathrm{F}$ & 6.903534000 & 6.205766000 & 2.283891000 \\
\hline $\mathrm{F}$ & 5.133532000 & 3.474661000 & I. 993404000 \\
\hline $\mathrm{F}$ & 6.292698000 & 4.502076000 & 3.690868000 \\
\hline $\mathrm{F}$ & 7.350339000 & 4.019247000 & І.697334000 \\
\hline $\mathrm{F}$ & 5.721062000 & 5.185658000 & 0.571892000 \\
\hline $\mathrm{F}$ & 4.675344000 & $5.6678 \mathrm{I} 6000$ & 2.556259000 \\
\hline $\mathrm{O}$ & 3.179542000 & 0.681348000 & 0.534471000 \\
\hline $\mathrm{H}$ & 3.926329000 & I.124827000 & 0.959523000 \\
\hline $\mathrm{C}$ & 3.620223000 & -0.544441000 & -0.022257000 \\
\hline $\mathrm{H}$ & 2.7332170000 & -I.068288000 & -o.38476ıоooo \\
\hline $\mathrm{H}$ & 4.112715000 & -I.157653000 & 0.738926000 \\
\hline $\mathrm{H}$ & 4.284842000 & -0.358791000 & -0.867783000 \\
\hline$P$ & 4.205204000 & -0.408635000 & 4.533378000 \\
\hline $\mathrm{F}$ & 5.729598000 & -0.934699000 & 4.620047000 \\
\hline $\mathrm{F}$ & 2.644273000 & 0.099534000 & 4.397409000 \\
\hline $\mathrm{F}$ & 3.940002000 & -0.795480000 & 6.074664000 \\
\hline $\mathrm{F}$ & 4.658858000 & I.088825000 & 4.932200000 \\
\hline $\mathrm{F}$ & 4.426320000 & -0.009503000 & 2.935084000 \\
\hline $\mathrm{F}$ & 3.7212890000 & $-\mathrm{I} .885535000$ & 4.041994000 \\
\hline \multicolumn{4}{|c|}{$S_{1} \cdot x y z$} \\
\hline \multicolumn{4}{|l|}{ II 8} \\
\hline \multicolumn{4}{|c|}{ symmetry cI } \\
\hline $\mathrm{Cu}$ & I.007634000 & I. 347627000 & 0.618226000 \\
\hline $\mathrm{Cu}$ & $-\mathrm{I} .457144000$ & I.937679000 & 0.314511000 \\
\hline $\mathrm{P}$ & o.8489640oo & 2.792027000 & $-\mathrm{I} .263836000$ \\
\hline $\mathrm{N}$ & -I.8582460oo & 2.988969000 & -I.345520000 \\
\hline $\mathrm{N}$ & -0.674132000 & 3.240203000 & -3.412175000 \\
\hline $\mathrm{C}$ & -0.763472000 & 2.999977000 & -2.143716000 \\
\hline
\end{tabular}




\begin{tabular}{|c|c|c|c|}
\hline $\mathrm{C}$ & -3.059502000 & 3.286825000 & -2.001961000 \\
\hline $\mathrm{H}$ & -3.959202000 & 3.274323000 & -I.409444000 \\
\hline $\mathrm{C}$ & -3.065046000 & 3.558731000 & -3.344061000 \\
\hline $\mathrm{H}$ & -4.001690000 & 3.786046000 & -3.843005000 \\
\hline $\mathrm{C}$ & -I.8631960oo & 3.517480000 & -4.061210000 \\
\hline $\mathrm{H}$ & -I.80535400o & 3.696224000 & -5.119278000 \\
\hline $\mathrm{C}$ & I. 304523000 & 4.498899000 & -0.886897000 \\
\hline $\mathrm{C}$ & 0.513378000 & 5.532936000 & -I.407063000 \\
\hline $\mathrm{H}$ & -0.369738000 & 5.313630000 & -I.994137000 \\
\hline $\mathrm{C}$ & 0.885767000 & 6.853084000 & $-\mathrm{I} .211894000$ \\
\hline $\mathrm{H}$ & 0.264154000 & 7.632536000 & -I.634342000 \\
\hline $\mathrm{C}$ & 2.042432000 & 7.155620000 & -0.500201000 \\
\hline $\mathrm{H}$ & 2.341877000 & 8.190456000 & -0.369608000 \\
\hline $\mathrm{C}$ & 2.818753000 & 6.133559000 & 0.043346000 \\
\hline $\mathrm{H}$ & 3.709571000 & 6.352868000 & 0.615169000 \\
\hline $\mathrm{C}$ & 2.454545000 & 4.804187000 & -0.145158000 \\
\hline $\mathrm{H}$ & 3.085027000 & 4.025909000 & 0.275019000 \\
\hline $\mathrm{C}$ & I.959007000 & 2.173830000 & -2.550887000 \\
\hline $\mathrm{C}$ & 2.633676000 & 3.070306000 & -3.386379000 \\
\hline $\mathrm{H}$ & 2.611435000 & 4.133477000 & -3.173698000 \\
\hline $\mathrm{C}$ & 3.309868000 & 2.594209000 & -4.505189000 \\
\hline $\mathrm{H}$ & 3.807897000 & 3.295715000 & -5.166844000 \\
\hline $\mathrm{C}$ & 3.335914000 & 1.225238000 & -4.774024000 \\
\hline $\mathrm{H}$ & 3.851663000 & 0.850651000 & -5.652350000 \\
\hline $\mathrm{C}$ & 2.677436000 & 0.330063000 & -3.930432000 \\
\hline $\mathrm{H}$ & 2.677600000 & -0.731450000 & -4.154414000 \\
\hline $\mathrm{C}$ & I.984873000 & 0.802869000 & -2.821134000 \\
\hline $\mathrm{H}$ & I.436927000 & o.1089380oo & -2.187734000 \\
\hline $\mathrm{P}$ & -I.712061000 & -0.326855000 & -0.118457000 \\
\hline $\mathrm{N}$ & 0.532417000 & -0.589685000 & I.300939000 \\
\hline $\mathrm{N}$ & -0.931126000 & -2.454256000 & I.441427000 \\
\hline $\mathrm{C}$ & -0.590967000 & $-\mathrm{I} .258443000$ & 0.989716000 \\
\hline $\mathrm{C}$ & I. 358335000 & -1.156353000 & 2.186042000 \\
\hline $\mathrm{H}$ & 2.272868000 & -0.629880000 & 2.417901000 \\
\hline C & 1.028152000 & -2.355517000 & 2.797532000 \\
\hline $\mathrm{H}$ & I.678883000 & -2.742091000 & 3.565293000 \\
\hline $\mathrm{C}$ & -0.118425000 & -2.998582000 & 2.356703000 \\
\hline $\mathrm{H}$ & -0.403720000 & -3.977751000 & 2.735719000 \\
\hline $\mathrm{C}$ & -I.213961000 & -0.818740000 & $-\mathrm{I} .783271000$ \\
\hline $\mathrm{C}$ & -0.428749000 & -I.9482IIOOO & -2.030507000 \\
\hline $\mathrm{H}$ & -0.178910000 & -2.609585000 & $-\mathrm{I} .209187000$ \\
\hline $\mathrm{C}$ & 0.013008000 & -2.213831000 & -3.322857000 \\
\hline $\mathrm{H}$ & 0.614631000 & -3.091690000 & -3.529072000 \\
\hline $\mathrm{C}$ & -0.318020000 & -I.347140000 & -4.360603000 \\
\hline
\end{tabular}




\begin{tabular}{|c|c|c|c|}
\hline $\mathrm{H}$ & 0.080462000 & -1.517090000 & -5.352374000 \\
\hline $\mathrm{C}$ & -I.1368780oo & -0.248948000 & -4.119718000 \\
\hline $\mathrm{H}$ & -I.398939000 & 0.436011000 & -4.914266000 \\
\hline $\mathrm{C}$ & $-I .594350000$ & o.01607900o & -2.837723000 \\
\hline $\mathrm{H}$ & -2.223926000 & 0.880857000 & -2.658642000 \\
\hline $\mathrm{C}$ & -3.345023000 & -I.0IOOI4000 & 0.200036000 \\
\hline $\mathrm{C}$ & -3.842769000 & -2.127330000 & -0.473106000 \\
\hline $\mathrm{H}$ & -3.236971000 & -2.626830000 & -I.221695000 \\
\hline $\mathrm{C}$ & -5.1122228000 & -2.596282000 & -0.163057000 \\
\hline $\mathrm{H}$ & -5.508060000 & -3.472398000 & -o.6664960oo \\
\hline $\mathrm{C}$ & -5.870658000 & -I.94056100o & 0.804125000 \\
\hline $\mathrm{H}$ & -6.854756000 & -2.307715000 & I.047713000 \\
\hline $\mathrm{C}$ & -5.370923000 & -0.836532000 & I. 484732000 \\
\hline $\mathrm{H}$ & -5.960875000 & -0.345457000 & 2.251372000 \\
\hline $\mathrm{C}$ & -4.100703000 & -0.370207000 & I.1835300oo \\
\hline $\mathrm{H}$ & -3.676154000 & 0.465982000 & I. 730720000 \\
\hline $\mathrm{P}$ & -І.088797000 & 3.077540000 & 2.248973000 \\
\hline $\mathrm{N}$ & I. 508473000 & 2.498763000 & 2.458268000 \\
\hline $\mathrm{N}$ & 0.822103000 & 4.504700000 & 3.544691000 \\
\hline $\mathrm{C}$ & 0.613709000 & 3.433097000 & 2.810161000 \\
\hline $\mathrm{C}$ & 2.718370000 & 2.608152000 & 3.022818000 \\
\hline $\mathrm{H}$ & 3.439926000 & $\mathrm{I} .83 \mathrm{I} 845000$ & 2.817861000 \\
\hline $\mathrm{C}$ & 3.040700000 & 3.687085000 & $3.83488 \mathrm{IOoo}$ \\
\hline $\mathrm{H}$ & 4.039122000 & 3.793281000 & 4.229801000 \\
\hline $\mathrm{C}$ & 2.068910000 & 4.654827000 & 4.017084000 \\
\hline $\mathrm{H}$ & 2.286377000 & 5.574952000 & 4.546453000 \\
\hline $\mathrm{C}$ & -2.004443000 & 4.616521000 & 2.389999000 \\
\hline $\mathrm{C}$ & -2.187767000 & 5.358174000 & I.217220000 \\
\hline $\mathrm{H}$ & -I.872373000 & 4.945813000 & 0.262161000 \\
\hline $\mathrm{C}$ & -2.761909000 & 6.623122000 & I.291273000 \\
\hline $\mathrm{H}$ & -2.874006000 & 7.2170009000 & 0.389142000 \\
\hline $\mathrm{C}$ & -3.191103000 & 7.118186000 & 2.519182000 \\
\hline $\mathrm{H}$ & -3.647450000 & 8.101517000 & 2.578312000 \\
\hline $\mathrm{C}$ & -3.040790000 & 6.359336000 & 3.680517000 \\
\hline $\mathrm{H}$ & -3.379940000 & 6.751856000 & 4.634538000 \\
\hline $\mathrm{C}$ & -2.416787000 & 5.120820000 & 3.629673000 \\
\hline $\mathrm{H}$ & -2.232288000 & 4.561599000 & 4.541807000 \\
\hline $\mathrm{C}$ & -I.712105000 & I. 858451000 & 3.450815000 \\
\hline $\mathrm{C}$ & -o.8693440oo & 0.817224000 & 3.869327000 \\
\hline $\mathrm{H}$ & 0.183836000 & 0.822078000 & 3.609005000 \\
\hline $\mathrm{C}$ & -I.367309000 & -0.206947000 & 4.663409000 \\
\hline $\mathrm{H}$ & -0.69822400o & -0.995185000 & 4.991319000 \\
\hline $\mathrm{C}$ & -2.707332000 & -0.216120000 & 5.046203000 \\
\hline $\mathrm{H}$ & -3.075842000 & -I.024795000 & 5.668574000 \\
\hline
\end{tabular}




\begin{tabular}{|c|c|c|c|}
\hline C & -3.549811000 & 0.813719000 & 4.630346000 \\
\hline $\mathrm{H}$ & -4.598270000 & 0.824253000 & 4.917400000 \\
\hline $\mathrm{C}$ & -3.059894000 & I. 845144000 & 3.833777000 \\
\hline $\mathrm{H}$ & -3.718954000 & 2.647486000 & 3.520344000 \\
\hline $\mathrm{P}$ & 6.015145000 & 4.846371000 & 2.125058000 \\
\hline $\mathrm{F}$ & 6.896352000 & 6.190647000 & 2.281544000 \\
\hline $\mathrm{F}$ & 5.111085000 & 3.469839000 & I.968663000 \\
\hline $\mathrm{F}$ & 6.262790000 & 4.485697000 & 3.678883000 \\
\hline $\mathrm{F}$ & 7.333657000 & 4.001640000 & I.694300000 \\
\hline $\mathrm{F}$ & $5 \cdot 724977000$ & 5.179927000 & 0.555285000 \\
\hline $\mathrm{F}$ & 4.662581000 & 5.662684000 & 2.530364000 \\
\hline $\mathrm{O}$ & 2.988176000 & 0.721750000 & 0.483759000 \\
\hline $\mathrm{H}$ & 3.700714000 & I.308479000 & 0.772182000 \\
\hline $\mathrm{C}$ & 3.534434000 & -0.471540000 & -0.067823000 \\
\hline $\mathrm{H}$ & 2.692934000 & -I.122090000 & -0.311556000 \\
\hline $\mathrm{H}$ & 4.166569000 & -0.960509000 & 0.677753000 \\
\hline $\mathrm{H}$ & 4.086769000 & -0.250201000 & -0.981601000 \\
\hline $\mathrm{P}$ & 4.177773000 & -0.419472000 & 4.528177000 \\
\hline $\mathrm{F}$ & 5.703969000 & -0.937490000 & 4.593614000 \\
\hline $\mathrm{F}$ & 2.609908000 & 0.085051000 & 4.413201000 \\
\hline $\mathrm{F}$ & 3.942288000 & -0.773814000 & 6.080859000 \\
\hline $\mathrm{F}$ & 4.633858000 & I.088373000 & 4.886908000 \\
\hline $\mathrm{F}$ & 4.359039000 & -0.052011000 & 2.916620000 \\
\hline $\mathrm{F}$ & 3.686098000 & $-\mathrm{I} .905089000$ & 4.076418000 \\
\hline \multicolumn{4}{|c|}{$\mathrm{T}_{\mathrm{I}} \cdot \mathrm{xyz}$} \\
\hline \multicolumn{4}{|l|}{ II 8} \\
\hline \multicolumn{4}{|c|}{ symmetry cI } \\
\hline $\mathrm{Cu}$ & I.016778073 & I. 348737095 & 0.613046043 \\
\hline $\mathrm{Cu}$ & -1.462345105 & I.940809140 & 0.304068022 \\
\hline $\mathrm{P}$ & 0.839780058 & 2.781460202 & $-\mathrm{I} .27334709 \mathrm{I}$ \\
\hline $\mathrm{N}$ & -I.897688137 & 2.943717213 & -I.353943098 \\
\hline $\mathrm{N}$ & -0.687206049 & 3.114606226 & -3.462892250 \\
\hline $\mathrm{C}$ & -0.726634051 & 2.868136207 & -2.123562154 \\
\hline $\mathrm{C}$ & -3.008725214 & 3.331977238 & -I.9883III43 \\
\hline $\mathrm{H}$ & -3.915846284 & $3.35976724 \mathrm{I}$ & -I.39735510I \\
\hline $\mathrm{C}$ & -3.034529220 & 3.645034260 & -3.328344240 \\
\hline $\mathrm{H}$ & -3.950701284 & 3.941661286 & -3.820270274 \\
\hline $\mathrm{C}$ & $-I .798555129$ & 3.481371252 & -4.039523289 \\
\hline $\mathrm{H}$ & $-\mathrm{I} .753141127$ & 3.6655730266 & -5.105011366 \\
\hline $\mathrm{C}$ & I. 332038097 & 4.486181325 & -0.885370064 \\
\hline $\mathrm{C}$ & 0.535475040 & 5.506979405 & -I.42330110I \\
\hline $\mathrm{H}$ & -0.339639024 & 5.260698376 & -2.015995146 \\
\hline $\mathrm{C}$ & 0.891312063 & 6.833742499 & -І.236761088 \\
\hline
\end{tabular}




\begin{tabular}{|c|c|c|c|}
\hline $\mathrm{H}$ & 0.272112019 & 7.607509547 & -I.674407120 \\
\hline $\mathrm{C}$ & 2.033940144 & 7.151477506 & -0.508273037 \\
\hline $\mathrm{H}$ & 2.320583167 & 8.190273613 & -0.378924027 \\
\hline $\mathrm{C}$ & $2.8 \mathrm{I} 400820 \mathrm{I}$ & 6.141238449 & 0.053970004 \\
\hline $\mathrm{H}$ & 3.694801263 & 6.375186441 & 0.636013045 \\
\hline $\mathrm{C}$ & 2.467584178 & 4.806408344 & -0.129881009 \\
\hline $\mathrm{H}$ & 3.099985223 & 4.036362291 & 0.302483022 \\
\hline $\mathrm{C}$ & I.9634IOI4I & 2.168009158 & -2.551537185 \\
\hline $\mathrm{C}$ & 2.653414194 & 3.066445223 & -3.373149244 \\
\hline $\mathrm{H}$ & 2.640568188 & 4.127617299 & -3.149028227 \\
\hline $\mathrm{C}$ & $3.32655924 \mathrm{I}$ & 2.597376186 & -4.496815322 \\
\hline $\mathrm{H}$ & 3.830566275 & 3.301782236 & -5.150815372 \\
\hline $\mathrm{C}$ & 3.343317242 & I. 230765087 & -4.778989343 \\
\hline $\mathrm{H}$ & 3.8582822277 & 0.860544063 & -5.659562426 \\
\hline $\mathrm{C}$ & 2.674896192 & 0.331948024 & -3.946329285 \\
\hline $\mathrm{H}$ & 2.669319193 & -0.727428055 & -4.180180300 \\
\hline $\mathrm{C}$ & I.977012140 & 0.799644059 & -2.839031205 \\
\hline $\mathrm{H}$ & I.4I $4254 \mathrm{IOI}$ & 0.106683008 & -2.218147157 \\
\hline $\mathrm{P}$ & $-\mathrm{I} .700029120$ & -0.318183023 & -0.131496009 \\
\hline $\mathrm{N}$ & 0.538979040 & -0.591709045 & I.29170809I \\
\hline $\mathrm{N}$ & -0.934724066 & $-2.449638 \mathrm{I} 74$ & I.429512102 \\
\hline $\mathrm{C}$ & -0.586383040 & -1.255148093 & 0.979960069 \\
\hline $\mathrm{C}$ & I.360341100 & -I.I62502084 & 2.178841157 \\
\hline $\mathrm{H}$ & 2.277382163 & -0.641129046 & 2.411730175 \\
\hline $\mathrm{C}$ & I.021668073 & -2.358970172 & 2.790918203 \\
\hline $\mathrm{H}$ & I.6673521I 8 & -2.748056195 & 3.561781259 \\
\hline $\mathrm{C}$ & -0.126518009 & -2.997300214 & 2.346958171 \\
\hline $\mathrm{H}$ & -0.416425030 & -3.975498287 & 2.724881195 \\
\hline $\mathrm{C}$ & -1.213193089 & -0.826400060 & -I.793809129 \\
\hline $\mathrm{C}$ & -0.421153031 & -I.952199140 & -2.032095147 \\
\hline $\mathrm{H}$ & -0.167642012 & -2.604928185 & $-\mathrm{I} .205208085$ \\
\hline C & 0.023578002 & -2.224569163 & -3.322432240 \\
\hline $\mathrm{H}$ & 0.628839047 & -3.100920221 & -3.522554254 \\
\hline $\mathrm{C}$ & -0.310007022 & -I.366I69098 & -4.365582312 \\
\hline $\mathrm{H}$ & 0.094336007 & -I.537096112 & -5.354606362 \\
\hline $\mathrm{C}$ & -I.138953083 & -0.273500020 & -4.134326298 \\
\hline $\mathrm{H}$ & -I.4OI284099 & 0.402995029 & -4.935659353 \\
\hline $\mathrm{C}$ & -I.598671II3 & -0.002411000 & -2.854648204 \\
\hline $\mathrm{H}$ & $-2.23729216 \mathrm{I}$ & 0.856290060 & -2.679353192 \\
\hline $\mathrm{C}$ & -3.337854238 & -0.991592073 & 0.192129014 \\
\hline $\mathrm{C}$ & -3.839072275 & $-2.10566415 \mathrm{I}$ & -0.484114035 \\
\hline $\mathrm{H}$ & -3.236303235 & -2.601269189 & $-\mathrm{I} .237602088$ \\
\hline $\mathrm{C}$ & -5.106398366 & -2.578065185 & -0.169921012 \\
\hline $\mathrm{H}$ & -5.503799420 & -3.451727250 & -0.676534047 \\
\hline
\end{tabular}




\begin{tabular}{|c|c|c|c|}
\hline $\mathrm{C}$ & -5.860171404 & -I.929504I4I & 0.805533056 \\
\hline $\mathrm{H}$ & -6.842918486 & -2.300057165 & I.051409073 \\
\hline $\mathrm{C}$ & -5.356388366 & -0.829379058 & I.490226105 \\
\hline $\mathrm{H}$ & -5.940876438 & -0.345168025 & 2.265464166 \\
\hline $\mathrm{C}$ & -4.087800293 & -0.359845026 & I.I85311084 \\
\hline $\mathrm{H}$ & -3.660138264 & 0.471052034 & I. 738519124 \\
\hline $\mathrm{P}$ & -I.109336o8o & 3.069108223 & 2.240805162 \\
\hline $\mathrm{N}$ & I.496310109 & 2.501027180 & 2.434292177 \\
\hline $\mathrm{N}$ & 0.803027057 & 4.485398323 & 3.552640256 \\
\hline $\mathrm{C}$ & 0.595797045 & 3.424340248 & 2.801735200 \\
\hline $\mathrm{C}$ & 2.707523197 & 2.607539188 & 2.997243216 \\
\hline $\mathrm{H}$ & 3.430938247 & I. 835925135 & 2.781818201 \\
\hline $\mathrm{C}$ & 3.028314218 & 3.676754266 & 3.821869273 \\
\hline $\mathrm{H}$ & 4.027273290 & 3.781520270 & 4.216292303 \\
\hline $\mathrm{C}$ & 2.051358146 & 4.635954336 & 4.020533287 \\
\hline $\mathrm{H}$ & 2.266207162 & 5.549575422 & 4.561933329 \\
\hline $\mathrm{C}$ & -2.012474143 & 4.616699332 & 2.395216173 \\
\hline $\mathrm{C}$ & -2.192578160 & 5.364525366 & I.226133087 \\
\hline $\mathrm{H}$ & -I.872018135 & 4.956512355 & 0.270631020 \\
\hline $\mathrm{C}$ & -2.762488198 & 6.631262456 & I.301938093 \\
\hline $\mathrm{H}$ & -2.869092208 & 7.229609521 & 0.401898029 \\
\hline $\mathrm{C}$ & -3.193155228 & 7.122950513 & $2.53066518 \mathrm{I}$ \\
\hline $\mathrm{H}$ & -3.647955260 & 8.106839559 & 2.592338185 \\
\hline $\mathrm{C}$ & -3.044811219 & 6.359822468 & 3.689298264 \\
\hline $\mathrm{H}$ & -3.383941243 & 6.749958500 & 4.644298334 \\
\hline $\mathrm{C}$ & -2.422846173 & 5.120081371 & 3.635244264 \\
\hline $\mathrm{H}$ & -2.240272159 & 4.557620328 & 4.545686328 \\
\hline $\mathrm{C}$ & $-\mathrm{I} .7258 \mathrm{III} 23$ & I. 858211133 & 3.458751247 \\
\hline $\mathrm{C}$ & -0.881569063 & 0.817496060 & 3.871199277 \\
\hline $\mathrm{H}$ & 0.170223012 & 0.821413062 & $3.60482326 \mathrm{I}$ \\
\hline $\mathrm{C}$ & $-\mathrm{I} .375529101$ & -0.208944015 & 4.666214337 \\
\hline $\mathrm{H}$ & -0.704401050 & -0.997642072 & $4.98907036 \mathrm{I}$ \\
\hline $\mathrm{C}$ & -2.713037197 & -0.219472016 & 5.055569362 \\
\hline $\mathrm{H}$ & -3.078069220 & -I.029236075 & 5.678439395 \\
\hline $\mathrm{C}$ & -3.556834256 & 0.810996059 & 4.645582335 \\
\hline $\mathrm{H}$ & -4.604197329 & 0.821813061 & 4.936959356 \\
\hline $\mathrm{C}$ & -3.070097223 & I. 844835135 & 3.849065279 \\
\hline $\mathrm{H}$ & -3.731652267 & 2.647179189 & 3.540066253 \\
\hline $\mathrm{P}$ & 6.025303440 & 4.851578348 & 2.133135155 \\
\hline $\mathrm{F}$ & 6.894743512 & 6.205352454 & 2.284651164 \\
\hline $\mathrm{F}$ & 5.136848368 & 3.466150251 & I. $98227614 \mathrm{I}$ \\
\hline $\mathrm{F}$ & 6.279749430 & 4.499256321 & 3.687917265 \\
\hline $\mathrm{F}$ & 7.353269513 & 4.020276292 & I.702814122 \\
\hline $\mathrm{F}$ & 5.729389428 & 5.179594375 & $0.56357904 \mathrm{I}$ \\
\hline
\end{tabular}




$\begin{array}{llcc}\text { F } & 4.665962337 & 5.656762390 & 2.538653185 \\ \text { O } & 2.996742218 & 0.711167052 & 0.466650034 \\ \text { H } & 3.715351269 & 1.286699090 & 0.761772055 \\ \text { C } & 3.532678256 & -0.487751035 & -0.080451006 \\ \text { H } & 2.685235193 & -1.124186079 & -0.340653025 \\ \text { H } & 4.147265301 & -0.990393074 & 0.671043049 \\ \text { H } & 4.102145293 & -0.270721019 & -0.984820070 \\ \text { P } & 4.174893301 & -0.411210030 & 4.518087327 \\ \text { F } & 5.700482432 & -0.931623066 & 4.586641329 \\ \text { F } & 2.608657186 & 0.096215007 & 4.400020315 \\ \text { F } & 3.938364284 & -0.760213057 & 6.071882415 \\ \text { F } & 4.633581336 & 1.097184080 & 4.872018349 \\ \text { F } & 4.358831312 & -0.049874004 & 2.905295209 \\ \text { F } & 3.681115263 & -1.897801137 & 4.071098295\end{array}$

